\title{
Estudo do escápulo-coracóide e da cartilagem sinarcual cérvico-torácica de Rhinoptera brasiliensis Müller \& Henle e Rhinoptera bonasus (Mitchill) (Elasmobranchii, Rhinopteridae)
}

\author{
Andreia Francisco Afonso ${ }^{1}$ \\ Valéria Gallo ${ }^{2}$
}

\begin{abstract}
Study of the scapulocoracoid and cervico-thoracic synarcual cartilage of Rhinoptera brasiliensis Müller \& Henle and Rhinoptera bonasus (Mitchill) (Elasmobranchii, Rhinopteridae). A comparative analysis of the scapulocoracoid and cervico-thoracic synarcual cartilage of juvenile specimens of Rhinoptera brasiliensis Müller \& Henle, 1841 and $R$. honasus (Mitchill, 1815) was accomplished. in order to identify specific, individual and ontogenetic differences. In the scapulocoracoid, the scapular foramen and the posterodorsal fenestra is larger in R. brasiliensis. The anterior fenestra bridge is thicker and the metacondyle is thinner and longer in $R$. honasus. This species also possesses a larger re-entrance between the mesocondyle and the metacondyle, as well as in the litting region of the synarcual cartilage, placed close to the scapular process. The individual differences are: posteroventral fenestra with variable diameter independent of the size of the specimens; scapulocoracoids, in some specimens, are more fragile even if they have been kept in the same conservation conditions. In the cervico-thoracic synarcual cartilage, the lateral stay in $R$. brasiliensis encloses completely the superior portion of the anteroscapular bridge. There are three more prominent condylcs beside the suprascapula in $R$. hrasiliensis. $R$. honasus, however, shows a protuberance close to the suprascapula, inconspicuous and more distant from the scapular lamina. Several specimens of different size of $R$. honasus show a synarcual cartilage wider in ventral view and higher in lateral view. In the majority of specimens, the number of ventral spinal foramina is higher than the dorsal ones in both species. $R$. honasus has only one pair of asymmetric basal foramina, whereas $R$. brasiliensis possesses three or four foramina. Regarding individual differences, at least one specimen of $R$. honasus exhibits a single basal foramen.

KEY WORDS. Chondrichthyes, Myliobatiformes, Rhinoplera, scapulocoracoid, cervico-thoracic synarcual cartilage, Brazil
\end{abstract}

$\Lambda$ cintura peitoral em Chondrichthyes, também denominada escápulo-coracóide, consiste de vários elementos anatômicos fusionados, suportando a nadadeira peitoral (LOVEJOY 1996). Ela está posicionada atrás da cabeça, como em outros Gnathostomata, localizando-se mais posteriormente em elasmobrânquios, não sendo ligada ao condroneurocrânio por elementos esqueléticos.

1) Departamento de Vertebrados, Museu Nacional, Universidade Federal do Rio de Janeiro. Quinta da Boa Vista, São Cristóvão, 20940-040 Rio de Janeiro, Rio de Janeiro, Brasil. E-mail: affa@terra.com.br

2) Departamento de Biologia Animal e Vegetal, Instituto de Biologia, Universidade do Estado do Rio de Janeiro. Rua São Francisco Xavier 524, Maracanā, 20559-900 Rio de Janeiro, Rio de Janeiro, Brasil. Bolsista Prociência UERJ/Faperj. E-mail: gallo@uerj.br 
Em raias, o escápulo-coracóide forma um anel orientado transversalmente no corpo, entre as cavidades branquial e abdominal, encontrando-se sempre em contato com a coluna vertebral. Segundo NiSHIDA (1990), esta estrutura apresenta uma forma em "U", compreendendo cartilagem coracóide, processo escapular e cartilagem supra-escapular, que se encontram fusionados.

A cartilagem sinarcual cérvico-torácica é uma peça alongada, composta por um conjunto de vértebras cervicais fusionadas, formando uma placa, característica de Batoidea dentre os Elasmobranchii, sendo também encontrada em Holocephali e † Placodermi. Esta estrutura articula-se anteriormente ao condroneurocrânio através do processo odontóide, que se encaixa em sua faceta articular mediana. Ventralmente, estão localizados os corpos vertebrais cérvico-torácicos. Estes são remanescentes da fusão parcial das vértebras, que estão situadas entre a porção posterior da supra-escápula e a porção final da cartilagem sinarcual, podendo variar individualmente (GOMES et al. 1997).

Tanto o escápulo-coracóide como a sinarcual vêm sendo utilizados por diversos autores em estudos sistemáticos de raias.

Compagno (1977) caracterizou a morfologia da superfïcie articular do escápulo-coracóide e suas relações com a cartilagem sinarcual como um todo e em maior detalhe com a supra-escápula, apontando diferenças entre as ordens, subordens e famílias de Batoidea.

MCEACHRAN \& COMPAGNO (1979) incluíram pela primeira vez o escápulo-coracóide em estudos de Rajoidei, em especial das famílias Gurgesiellidae, Rajidae e Pseudorajidae, e demonstraram a importância desta estrutura anatômica no contexto taxonômico e filogenético. Estes autores propuseram algumas hipóteses filogenéticas para os Rajoidei, utilizando a morfologia e uma série de medições da parte lateral do escápulo-coracóide, o que permitiu o reconhecimento de gêneros e subgêneros.

MCEACHRAN (1983) verificou dimorfismo sexual no escápulo-coracóide de Psammobatis Gunther, 1870. Nas fềmeas, ele é mais retangular e há uma distância maior entre o mesocôndilo e o metacôndilo, em relação aos machos. O mesmo autor comparou o escápulo-coracóide nas espécies Bathyraja asperula Garrick \& Paul, 1974 e B. spinifera Garrick \& Paul, 1974, observando algumas diferenças principalmente em relação à forma, ao diâmetro da fenestra póstero-dorsal e ao número de fenestras póstero-ventrais (MCEACHRAN 1984).

LEIBLE (1988) descreveu o escápulo-coracóide de raias como tendo uma forma retangular, com bordas dorsal, ventral, anterior e posterior. Além disso, utilizou a metodologia de MCEACHRAN \& COMPAGNO (1979) e MCEACHRAN (1983) para efetuar medições da parte lateral desta estrutura.

MCEACHRAN \& MIYAKE (1990) utilizaram, dentre outras estruturas, o escápulo-coracóide para propor uma fillogenia dos Rajoidei. Segundo os autores, a ponte anterior delgada ou ausente e a presença de fenestra póstero-dorsal única são sinapomorfias deste grupo.

NiSHIDA (1990), em seu estudosobre a filogenia da subordem Myliobatidoidei, comparou o escápulo-coracóide de vários gêneros de raias e apresentou uma descrição 
geral da cartilagem sinarcual. O autor utilizou, ainda, uma nova terminologia para as diferentes regiões da coluna vertebral: primeira cartilagem sinarcual (correspondente à cartilagem sinarcual cérvico-torácica, sensu C.OMPAGNO 1973); vértebras intersinarcuais; segunda sinarcual (correspondente à cartilagem sinarcual tóraco-lombar, sensu ComPAGNO 1973); vértebras monospondílicas e diplospondílicas.

BRITO \& SERET (1996) propuseram o gênero Iansan para o "Rhinobatidae" fössil, Rhinobatos beurleni Silva Santos, 1968, com base em complexos anatômicos, dentre eles o escápulo-coracóide, que foram considerados pelos autores relevantes para a identificação e estudos fillogenéticos.

Outros autores, ainda, descreveram a cartilagem sinarcual cérvico-torácica de diversas espécies de raias, no contexto de trabalhos sistemáticos (e.g. GARMAN 1913; Daniel 1934; Melouk 1949; Saint-Seine 1949; Silva Santos 1968; NiSHIDA 1990; MAISEY 1991). Apenas GOMES et al. (1997) realizaram um detalhado estudo comparativo desta estrutura em Rajídeos.

No presente trabalho, realizou-se a primeira análise comparativa do escápulo-coracóide e da cartilagem sinarcual cérvico-torácica em Rhinoptera Cuvier, 1829, estruturas estas que se mostraram válidas na diferenciação taxonômica das duas espécies estudadas. Outros cstudos comparativos entre Rhinoptera brasiliensis Müller \& Henle, 1841 e $R$. bonasus (Mitchill, 1815) incluem a anatomia do condroneurocrânio (GALL.O-DA-SiLv^ el cl. 1997) e sua discriminação estatística com base em caracteres morfométricos externos (CAVALCANTI et al. 1997).

\section{MATERIAL E MÉTODOS}

O material zoológico utilizado foi proveniente das localidades de Pedra e Barra de Guaratiba, praia de Copacabana e Baia de Sepetiba, todas na cidade do Rio de Janeiro, estado do Rio de Janeiro, Brasil. Os animais foram identificados inicialmente pela dentição, segundo BigELow \& SCHROEDER (1953).

Os espécimens foram preparados para a observação do escápulo-coracóide e da cartilagem sinarcual cérvico-torácica, sendo aquecidos em água, e adicionando-se uma pequena quantidade de carbonato de potássio calcinado, o que facilita a soltura da carne. Após a limpeza do esqueleto em água corrente, colocou-se parte do material anatômico em álcool $70 \%$ e parte em uma solução de formaldeído a $10 \%$.

Os exemplares, após sua preparação, foram catalogados na Coleção Anatômica de Chondrichthyes do Departamento de Biologia Animal e Vegetal do Instituto de Biologia da Universidade do Estado do Rio de Janeiro (AC.DBAV.UERJ).

Por questões de disponibilidade amostral, foram considerados apenas exemplares jovens, entendendo-se como tal aqueles que não atingem $2000 \mathrm{~mm}$ de largura do disco. Segundo Bigelow \& SCHROEDER (1953), esta seria aproximadamente a medida encontrada em exemplares adultos de Rhinoptera.

Para a contagem dos forâmens espinais da raiz dorsal e ventral, a cartilagem sinarcual foi colocada na posição comumente encontrada na literatura (vide NISHIDA 1990; LOVEJOY 1996). Na região de contato entre as pontes ântero- e póstero-escapulares, houve uma interrupção da contagem, pois os forâmens estão cobertos pela mesma, sendo novamente contados após esta região. 
A terminologia anatômica e dados morfométricos foram baseados nos trabalhos de Compagno (1973), McEachran \& COMPagno (1979), McEACHran (1984), Leible (1988), Nishida \& Nakaya (1988), McEachran \& MiYake (1990), NishidA (1990) e GOMES et al. (1997).

As descrições anatômicas foram efetuadas a partir de $R$. brasiliensis, comparando-as com aquelas de $R$. honasus. Seguindo-se este critério, apenas nas liguras referentes à $R$. brasiliensis as estruturas foram indicadas. Todas as liguras apresentam uma seta apontando para a região anterior do corpo.

Abaixo é apresentada a lista dos exemplares das espécies examinadas, onde são representados, respectivamente, o número da coleção e, quando possivel, o sexo, o comprimento total (CT), a largura do disco (LD) e a procedência:

Rhinoptera brasiliensis: AC.DBAV.UERJ. 709, fềmea, $573 \mathrm{~mm} \mathrm{CT,} 540 \mathrm{~mm}$ LD, Barra de Guaratiba; AC.DBAV.UERJ. 770, fềmea, $857 \mathrm{~mm} \mathrm{CT,} 650 \mathrm{~mm}$ LD, procedência indeterminada; AC.DBAV.UERJ. 851, fềmea, 746mm CT, $605 \mathrm{~mm}$ LD, praia de Copacabana; AC.DBA V.UERJ. 887, macho, 552mm CT, LD e procedência indeterminados; AC.DBA V.UERJ. 906, sexo, CT, LD e procedência indeterminados; e AC.DBA V.UERJ. 914, 422mm LD, sexo, CT e procedência indeterminados.

Rhinoptera bonasus: AC.DBA V.UERJ. 068, macho, 782mm CT, 438mm LD, Pedra de Guaratiba; AC.DBA V.UERJ. 338, fềmea, 694mm CT, 466mm LD, Pedra de Guaratiba; AC.DBAV.UERJ. 886, sexo, CT e LD indeterminados, praia de Copacabana; AC.DBA V.UERJ. 917, macho, 707mm CT, 852mm LD, Baia de Sepetiba; AC.DBA V.UERJ. 919, macho, 613mm CT, 609mm LD, Baía de Sepetiba; AC.DBA V.UERJ. 926, macho, $881 \mathrm{~mm}$ CT, $715 \mathrm{~mm}$ LD, Baía de Sepetiba.

\section{RESULTADOS}

\section{Descrição}

O escápulo-coracóide, em vista dorsal (Figs l-2), exibe, em sua porção posterior, o processo escapular (Fig. 1 PE), que se articula firmemente com a face lateral da cartilagem sinarcual cérvico-torácica. Existe uma depressão localizada entre o processo escapular e a ponte escapular (Fig. I PS), denominada depressão escapular (Fig. I DE).

Em vista lateral, o escápulo-coracóide apresenta forma trapezoidal (Figs 3-4), podendo-se observar também a presença de forâmens e fenestras, para a passagem de artérias, nervos e/ou ancoragem de músculos, e côndilos para os pterigios peitorais. O procôndilo (Fig. 3 PRC) (para o propterígio), mesocôndilo (Fig. 3 MSC) (para o mesopterígio) e metacôndilo (Fig. 3 MTC) (para o metapterígio) estão dispostos ao longo do eixo horizontal desta estrutura.

Nas espécies aqui estudadas, há apenas um mesocôndilo (Figs 3-4). Com relação ao metacôndilo, este é mais estreito ligeiramente mais longo em $R$. bonasus (cerca de $0,7 \%$ LD) do que em $R$. brasiliensis (cerca de $0,5 \%$ LD).

Ocorrem dois pares de lenestras completamente separados: as fenestras ântero- (Fig. 3 FAD) e póstero-dorsais (Fig. 3 FPD), que se localizam acima dos côndilos e as fenestras ântero- (Fig. 3 FAV) e póstero-ventrais (Fig. 3 FPV), localizadas abaixo dos mesmos. Nos exemplares com maior largura de disco, a 
fenestra póstero-dorsal apresenta maior diâmetro em $R$. brasiliensis (cerca de $0,22 \%$ LD) do que em $R$. honasus (cerca de $0,02 \%$ LD). Já nos excmplares menores, o tamanho desta fenestra se assemelha nas duas espécies.
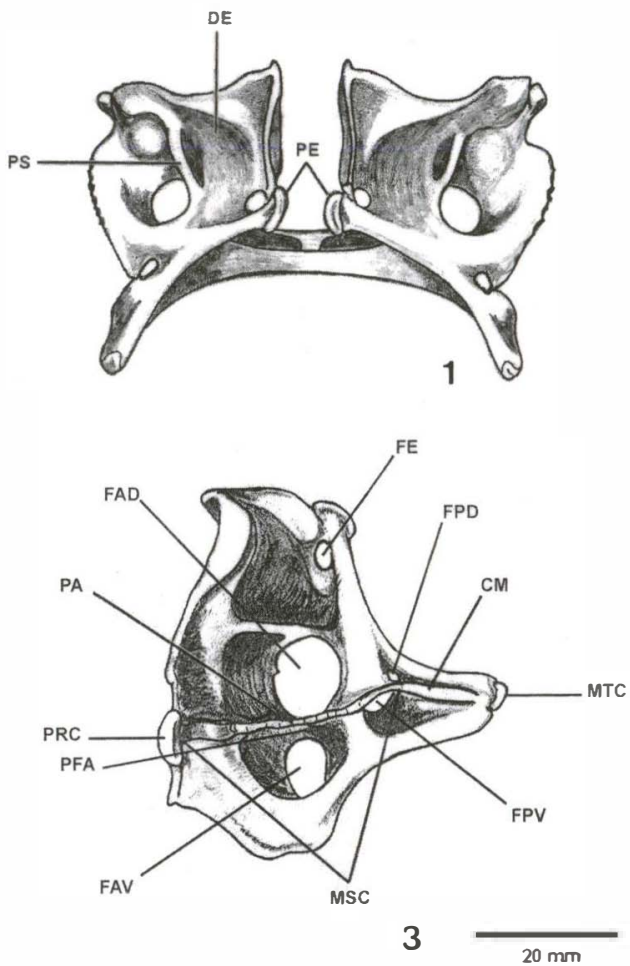
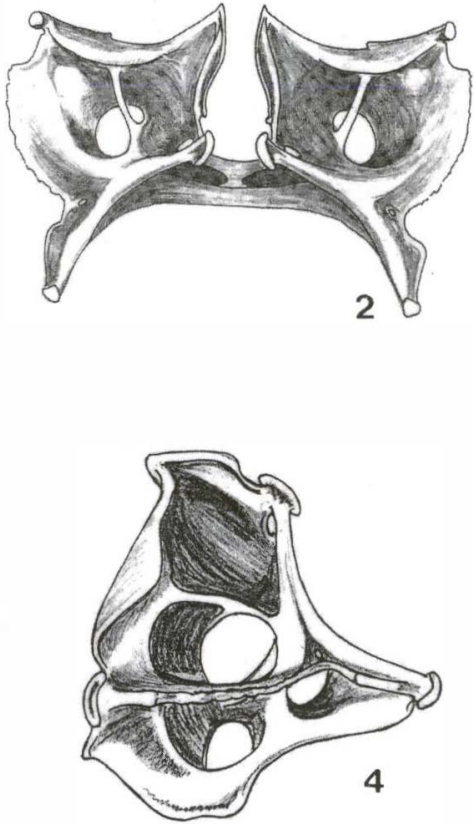

Figs 1-4. Representação do escápulo-coracóide. (1) Rhinoptera brasiliensis, AC.DBAV.UERJ. 709 e 887, vista dorsal; (2) $R$. bonasus, AC.DBAV.UERJ. 919 e 926, vista dorsal; (3) $R$. brasiliensis, AC.DBAV.UERJ. 709 e 887, vista lateral; (4) R. bonasus, AC.DBAV.UERJ. 919 e 926, vista lateral. (CM) Crista mesopterigiana, (DE) depressão escapular, (FAD) fenestra ântero-dorsal, (FAV) fenestra ântero-ventral, (FE) forâmen escapular, (FPD) fenestra póstero-dorsal, (FPV) fenestra póstero-ventral, (MSC) mesocôndilo, (MTC) metacôndilo, (PA) ponte anterior, (PE) processo escapular, (PFA) ponte das fenestras anteriores, (PRC) procôndilo, (PS) ponte escapular.

Entre o pró- e o mesocôndilo estende-se a ponte anterior (Fig. 3 P $\Lambda$ ), e entre o meso- c o metacôndilo se esboça a crista mesopterigiana (Fig. 3 CM). Dorsal- e ventralmente à ponte anterior, abrcm-se as fenestras ântero-dorsal e ântero-ventral. Dorsalmente à crista mesopterigiana, situa-se a pequena fenestra póstero-dorsal e ventralmente a cla, a grande fenestra póstero-ventral. Este conjunto de aberturas dá passagem a vasos sangüineos, nervos e músculos. As fenestras provavelmente atuam também no alívio de tensão dos músculos da região peitoral durante a locomoção do animal. 
Na facc interna do escápulo-coracóide, verificou-se a presença de dois côndilos para a articulação do quinto epibranquial e ceratobranquial. Segundo NiSHIDA (1990), estes côndilos são proximamente localizados em muitos Myliobatidoidei, porém muito separados em Rhinoptera. Não foi verificada diferenç̧a considerável na distância entre os dois côndilos para as espécies de Rhinoptera aqui estudadas.

O forâmen escapular (Fig. 3 FE), localizado próximo ao processo escapular, apresenta diâmetro cerca de duas vezes e meia maior em $R$. brasiliensis do que em $R$. bonasus (Figs 3-4).

A ponte das fenestras anteriores (Fig. 3 PFA) é mais espessa em $R$. bonasus (Figs 3-4). Também nesta espécie, observa-se uma maior reentrância entre o mesocôndilo e o metacôndilo, assim como na região de encaixe da cartilagem sinarcual, localizada próximo ao processo escapular.

Considerando o aspecto geral da cartilagem sinarcual, nota-se que, em $R$. bonasus, ela é ligeiramente mais alta (cerca de 5\% LD) do que em $R$. brasiliensis (cerca de $4,5 \% \mathrm{LD}$ ), quando vista lateralmente, e mais larga, quando observada ventralmente.

A extremidade anterior da cartilagem sinarcual é delimitada pelo processo odontóide (Fig. 5 PO), que se apresenta bifurcado. Este processo se articula com a porção posterior do condroneurocrânio, à frente dos côndilos occipitais, sendo a articulação continua com o primeiro par de extensões laterais (Fig. 5 EL). Estas são projeções que se estendem ântero-posteriormente por quase todo o comprimento da sinarcual, estando o primeiro par localizado logo a seguir ao processo odontóide e o último par próximo à ponte ântero-escapular. Posteriormente ao processo odontóide, localiza-se o colar occipital (Fig. 5 CO), de formato arredondado.

Os forâmens basais (Fig. 5 FB) estão localizados na região basal da cartilagem sinarcual, abaixo do processo odontóidc, posicionando-se em relação à primeira, entre a primeira e a segunda, ou em relação à segunda extensão lateral. Em $R$. brasiliensis, com exceção do exemplar AC.DBAV.UERJ. 914, que apresenta dois forâmens basais, ocorre sempre mais de um par destes forâmens (Fig. 5), podendo variar de três a quatro. Já em $R$. honasus, há sempre um par (Fig. 6), exccto o exemplar AC.DBA V.UERJ. 926, com um único forâmen basal. Observou-se uma variação na posição do forâmens. Na maioria dos exemplares de $R$. bonasus, os forâmens não estão arranjados simetricamente, ou seja, eles estão situados diagonalmente um em relação ao outro, estando, em geral, posicionados entre a primeira e a segunda extensão lateral. Em $R$. brasiliensis, os forâmens basais também mostram uma assimetria, porém bem menos acentuada que em $R$. bonasus. Nos exemplares com dois pares de forâmens, estes situam-se bem próximos e não ultrapassam o nivel basal da primeira extensão lateral. Naqueles com três forâmens, há uma maior variação, podendo localizar-se na primeira, entre a primeira e a segunda, ou na segunda extensão lateral.

O septo intermuscular anterior (Fig. 7 SIA) situa-se dorsalmente, estendendo-se do processo odontóide à supra-escápula (Fig. $7 \mathrm{SE}$ ).

O suporte lateral (Fig. $9 \mathrm{SL}$ ) é uma cstrutura cordiforme, que se localiza abaixo do septo intermuscular anterior e encontra-se fusionado, total- ou parcialmente, à ponte ântero-escapular (Fig. 10 PAE). Em R. brasiliensis, não é possível 
observar a porção superior da ponte, já que o suporte latcral a encobrc totalmente. $\mathrm{O}$ mesmo não acontece com $R$. bonasus, sendo a porção superior da ponte visível em vista lateral (Fig. 10).
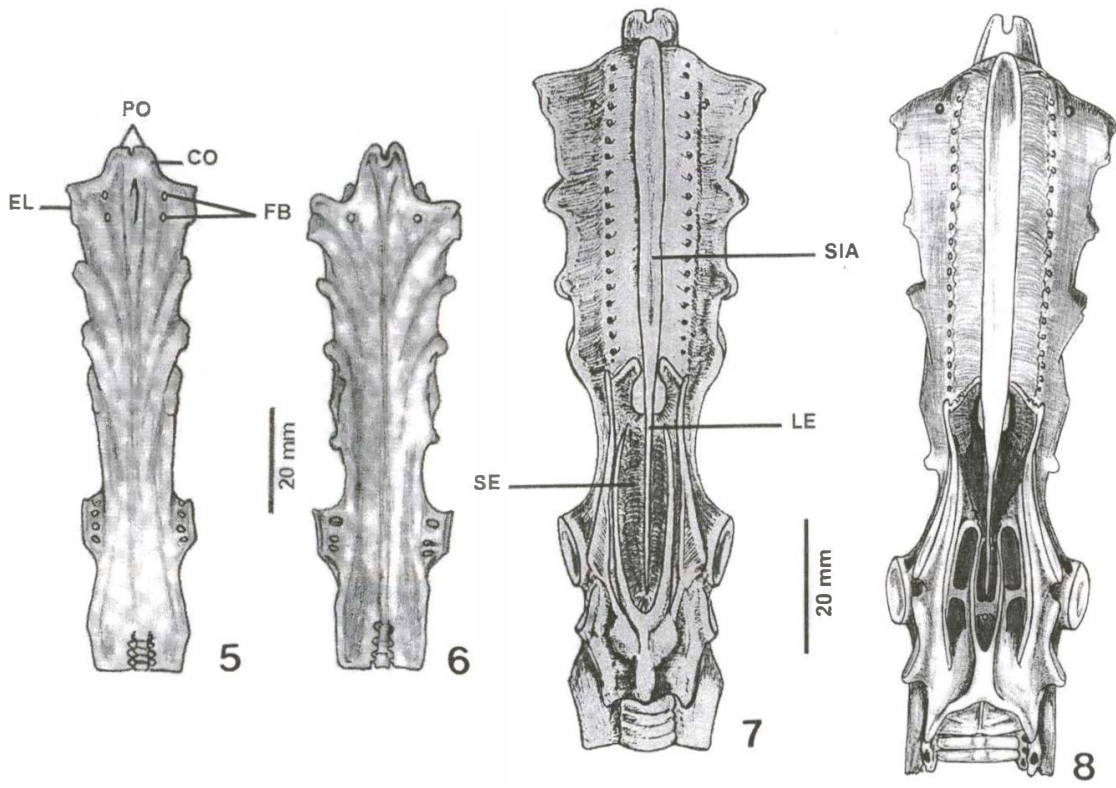

Figs 5-8. Representação da cartilagem sinarcual cérvico-torácica. (5) Rhinoptera brasiliensis, AC.DBAV.UERJ. 851 e 887, vista ventral; (6) R. bonasus, AC.DBAV.UERJ. 926, vista ventral; (7) $R$. brasiliensis, AC.DBAV.UERJ. 851 e 887, vista dorsal; (8) $R$. bonasus, AC.DBAV.UERJ. 926, vista dorsal. (CO) Colar occipital, (EL) extensão lateral, (FB) forâmens basais; (LE) lâmina escapular, (PO) processo odontóide, (SE) supra-escápula, (SIA) septo intermuscular anterior.

A supra-escápula (Fig. 7 SE) encontra-se fusionada à cartilagem sinarcual e localiza-se acima da superfície articular para o processo escapular (Fig. 9 S^P). Em $R$. brasiliensis, a supra-escápula é mais compacta; porém em $R$. boncisus, é mais curta e expandida, pois as cartilagens que a delimitam lateralmente tendem a se afastar do centro desta região (Figs 7-8). Posteriormente, notam-se três côndilos mais protuberantes em $R$. brasiliensis. No centro da supra-escápula, percebe-se uma cartilagem em forma de lâmina, denominada lâmina escapular (Figs 7, 9 LE), sendo esta mais alta em $R$. bonasus, porém mais comprida em $R$. brasiliensis. Posteriormente à supra-escápula, $R$. bonasus apresenta uma protuberância pouco conspicua e bem afastada da lâmina escapular.

Devido à continuidade existente entre a porção póstero-lateral da supra-cscápula e as pontes ântero-escapular (Fig. 10 PAE) e póstero-escapular(Fig. 9 POE), há a formação de uma grande fenestra, onde dorsalmente localiza-se a supcrfície articular para o processo escapular. O diâmetro desta fenestra não varia consideravelmente em ambas as espécies. 


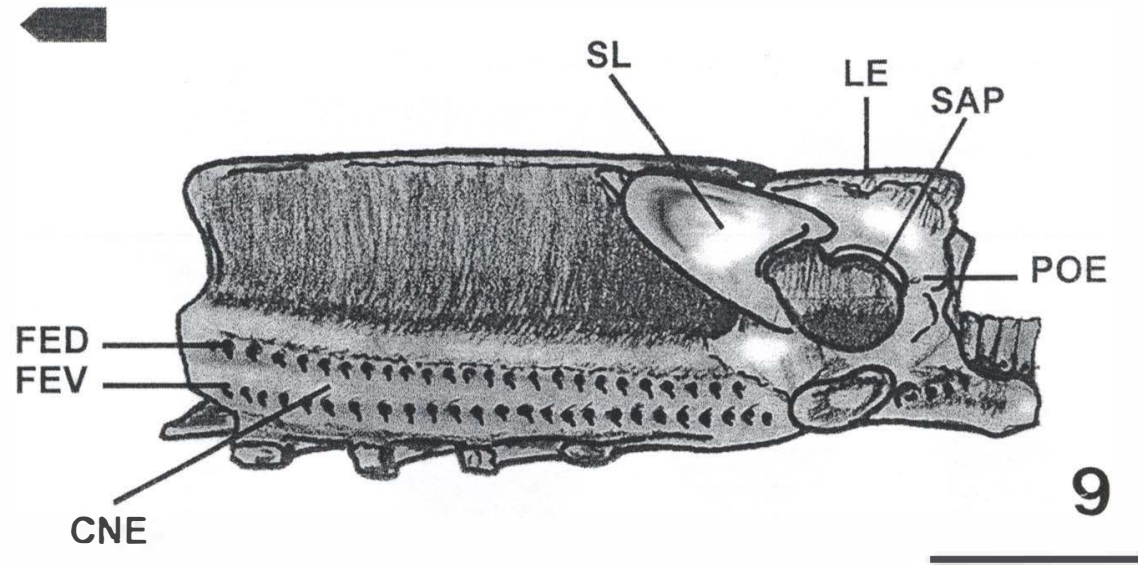

$20 \mathrm{~mm}$

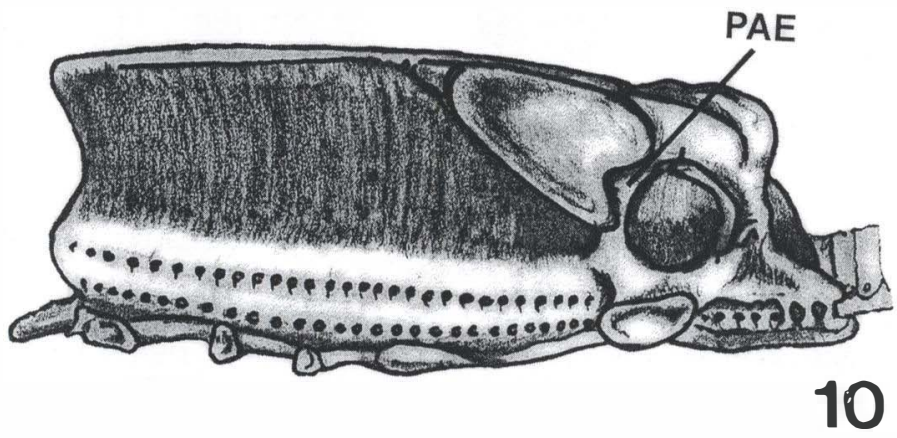

Figs 9-10. Representação da cartilagem sinarcual cérvico-torácica (9) Rhinoptera brasiliensis, AC.DBAV.UERJ. 851 e 887, vista lateral; (10) R. bonasus, AC.DBAV.UERJ. 926, vista lateral. (CNE) Canal dos nervos espinais, (FED) forâmen espinal da raiz dorsal, (FEV) forâmen espinal da raiz ventral, (LE) lâmina escapular, (PAE) ponte ântero-escapular, (POE) ponte pósteroescapular, (SAP) superficie articular para o processo escapular, (SL) suporte lateral.

Os forâmens espinais da raiz dorsal (Fig. 9 FED) e ventral (Fig. 9 FEV) iniciam-se após o processo odontóide e estão alinhados em duas fileiras horizontais, próximo à base da cartilagem sinarcual. Entre as duas fileiras de forâmens espinais, encontra-se o canal para os nervos espinais (Fig. 9 CNE). Ventralmente à fenestra, os forâmens espinais são encobertos pela estrutura resultante da união das pontes ântero- e póstero-escapulares. Somente após esta estrutura, os forâmens voltam a ser observados e contados (Tab. I), já que continuam enfileirados de uma extremidade a outra da cartilagem sinarcual (Fig. 9). Com exceção do espécimen AC.DBAV.UERJ. 709, os demais apresentam um número maior de forâmens espinais ventrais. Em vista ventral, existem várias aberturas localizadas na extensão lateral mais posterior, que se comunicam com os forâmens espinais da raiz ventral. 
Tabela I. Número de forâmens espinais das raizes dorsal e ventral.

\begin{tabular}{ccc}
\hline & \multicolumn{2}{c}{ Forâmens espinais das raizes } \\
\cline { 2 - 3 } Espécimens & Dorsal & Ventral \\
\hline Rhinoptera brasiliensis & & \\
AC.DBAV.UERJ. 709 & 33 & 33 \\
AC.DBAV.UERJ. 770 & 28 & 38 \\
AC.DBAV.UERJ. 851 & 31 & 39 \\
AC.DBAV.UERJ. 887 & 30 & 39 \\
AC.DBAV.UERJ. 914 & $28^{*}$ & 37 \\
Rhinoptera bonasus & & 32 \\
AC.DBAV.UERJ. 068 & 27 & 35 \\
AC.DBAV.UERJ. 338 & 27 & 38 \\
AC.DBAV.UERJ. 886 & 28 & 37 \\
AC.DBAV.UERJ. 917 & 28 & 36 \\
AC.DBAV.UERJ. 919 & 29 & 40 \\
AC.DBAV.UERJ. 926 & 29 & \\
\hline
\end{tabular}

(*) Contagens realizadas no lado esquerdo da sinarcual, exceto no exemplar AC.DBAV.UERJ. 914, cuja região da face lateral esquerda, onde se localizam os forâmens, encontra-se fragmentada.

\section{DISCUSSÃO}

Com relação à cintura peitoral, as raias são bastante distintas dos tubarões, uma vez que nestes esta estrutura é totalmente independente da coluna vertebral. Cada porção individualizada da cintura é composta ventralmente pelo coracóide e dorsalmente pela escápula. Nas raias, as porções direita e esquerda da cintura são unidas dorsalmente por meio das cartilagens supra-escapulares. Com exceção dos Torpedinidae, que apresentam um padrão de articulação intermediário entre tubarões e raias (DANIEL 1934), essas cartilagens estão firmemente unidas à cartilagem sinarcual, que em sua parte mediana e dorsal formam a supra-escápula (REG^N 1906).

COMPAGNO (1973) propõe que a união do escápulo-coracóide com a coluna vertebral, assim como a presença da cartilagem sinarcual cérvico-torácica, estariam relacionadas ao tipo de locomoção das raias, que se dá por movimentos ondulatórios das nadadeiras peitorais, principalmente nos Myliobatidoidei, cuja cauda é bastante delgada. Além disso, a presença de uma segunda cartilagem sinarcual neste grupo ("cartilagem sinarcual tóraco-lombar", sensu COMPAGNO 1973) estaria associada a uma maior rigidez da coluna vertebral. De acordo com LEIBLE (1988), nas raias que possuem nadadeiras peitorais pequenas (alguns "Rhinobatidae" e Pristidae), a união com a coluna vertebral é mais fraca e menos complexa, e a cintura mais simples do que em raias com nadadeiras peitorais desenvolvidas, como os Myliobatidoidei.

O escápulo-coracóide em alguns Myliobatidoidei apresenta um formato subretangular a trapezoidal, em vista lateral, sendo caracteristicamente trapezoidal em Rhinoptera. Em Gymnura Kuhl \& Van Hasselt, 1823 e Aetoplatea Müller \& Henle, 184 I apresenta-se alongado ântero-posteriormente. Já em Mohula Rafines- 
que, 1810 e Manta Bancroft, 1828, a estrutura é alongada anteriormente c fusiona-se ao proptcrígio peitoral (NISHIDA 1990). Segundo MCEACHRAN \& COMPAGNO (1979), a face lateral do escápulo-coracóide de Gurgesiella furvescens De Buen, 1959, assim como de muitos Rajoidei, é alongada ântero-posteriormente, o mesmo ocorrendo entre os Myliobatidoidei.

Em Rhinoptera, o escápulo-coracóide apresenta quatro fenestras bem individualizadas: duas anteriores (uma ventral e uma dorsal) e duas posteriores (uma ventral e outra dorsal). As pontes das fenestras anteriores situam-se na face interna do escápulo-coracóide, sendo uma característica diagnóstica do gênero estudado. Segundo NiSHIDA (1990), a condição e o número de fenestras ântero-dorsais variam, sendo observada fenestra única em alguns gêneros (e.g. Potamortygon Garman, 1877, Gymnura e Aetoplatea) ou duas fenestras completamente separadas (e.g. Dasyatis Rafinesquc, 1810 e Himantura Müller \& Henle, 1837). Além disso, em Dasvatis, a depressão escapular possui estreita comunicação com a fenestra ântero-dorsal. A fenestra póstero-dorsal está presente na maioria dos Myliobatidoidei, não ocorrendo em Urolophus Müller \& Henle, 1837, na maioria das espécies de Urotrygon Gill, 1864 e em Rhinoptera javanica Müller \& Henle, 1841 (NisHidA 1990).

MCEACHRAN \& MIYAKE (1990) alirmam que a presença de uma ponte anterior espessa e mais de uma fenestra póstero-dorsal no escápulo-coracóide são consideradas condições plesiomórficas. Entretanto, NisHidA (1990) e LoveJOY (1996) sugerem que, de um modo geral, a presença de fenestras e forâmens é considerada um estado derivado. Da mesma forma, MCEACHRAN \& MIYAKE (1990) apontam uma condição apomórfica para o processo escapular longo e posteriormente deslocado presente em Torpediniformes.

Em algumas formas recentes de "Rhinobatidae" e Pristidae, o escápulo-coracóide é muito alto e estreito, como em tubarões, apresentando três côndilos articulares para as estreitas cartilagens basais peitorais. Squatina Duméril, 1806 tem dois côndilos que são orientados diagonalmente, um para o propterigio c mesopterígio, e outro para o metapterígio (SHIRAI 1992). A orientação horizontal dos côndilos, tal como ocorre em Rhinoptera, segundo MCEACHRAN \& MIYAKE(1990), é considerada um estado derivado. Na maioria das raias, o mesocôndilo é uma estrutura única como aqui verificado para Rhinoptera. Porém, segundo NiSHidA (1990), em Myliobatidoidei o número de mesocôndilos pode variar de zero a quatro. Em Rhinohatos Linck, 1790, Zapteryx Jordan \& Gilbert 1880, Trygonorrhina Müller \& Henle, 1841 e Rajoidei, o escápulo-coracóide é distintamente alongado entre o mesocôndilo e o metacôndilo (MCEACHRAN \& MIYAKE 1990).

$\Lambda$ união sinarcual-escapular dos Myliobatidoidei, com os processos escapulares articulando-se com as faces laterais da sinarcual (articulação "ball-and-socket", sensu MCEACHRAN et al. 1996) e as supra-escápulas fusionando-se com ambos os lados da sinarcual, poderia ser derivada de um encaixe de juntas supraescapular/escapular de "Rhinobatidae" recentes (COMPAGNO 1973).

HOLMGREN $(1940,1942)$ demonstrou que a cartilagem sinarcual em raias é formada ontogeneticamente a partir de um espaço existente entre a porção anterior da coluna vertebral e a região posterior do condroneurocrânio. Esta estrutura está presente, 
ainda, em placodermas e holocéfalos, o que levou alguns autores no passado a propor que os holocéfalos tinham herdado esta estrutura de um "ancestral placoderma" (e.g. ØrVIG 1962; PATTERSON 1965). Esta idéia foi reforçada por STENSiö (1969) que considerou placodermas um grupo parafilético, a partir do qual teriam se originado independentemente vários grupos de Chondrichthyes. Entretanto, JANVIER (1996) sustenta um monofiletismo para os † Placodermi, sendo este provavelmente grupo-irmão de Chondrichthyes. De qualquer modo, a presença de sinarcual em placodermas, raias e quimeras deve ter sido adquirida independentemente.

Para Lund \& Grogan (1997), a placa basal fusiona-se com os elementos anteriorcs da coluna vertebral para dar origem à sinarcual em Helodus $\Lambda$ gassiz, 1838, Chimaeridae e alguns Cochliodontidae. Esta estrutura parece funcionar como um suporte para a musculatura atuante na mobilidade dos espinhos cranianos e da nadadeira dorsal.

GARMAN (1913) observou um crescimento gradual do número de vértebras fusionadas à cartilagem sinarcual a partir dos grupos mais basais ("Rhinobatiformes" e Pristiformes) até as formas mais apicais (Myliobatiformes). Este último grupo é caracterizado ainda pela duplicação da sinarcual, aumentando assim a área de fusão vertebral (GOMES et cl. 1997).

Da mesma forma, MAISEY (1984) afirmou que em raias do Jurássico (e.g. Beleminobatis Thiollière, 1854 e Spathohatis Thiollière, 1854) a sinarcual é muito curta, ou seja, possui um pequeno número de vértebras fusionadas. Com base nesta afirmativa, MCEACHRAN \& MIYAKE (1990) propuseram ser esta uma condição primitiva em "Rhinobatiformes", Pristiformes e Torpediniformes, e derivada nas demais raias.

Melouk (1949) apontou a presença de cerca de 20 vértebras incluidas na carti lagem sinarcual em Torpedo e 39 vértebras em Myliobatis. Nos exemplares de Rhinoptera aqui estudados, o número de vértebras variou de 27 a 40.

A presença de uma larga fenestra resultante da união da porção póstero-lateral da supra-escápula com as pontes ântero-escapular e póstero-escapular em Rhinoptera brasiliensis e $R$. bonasus não é um caráter amplamente distribuido dentro dos Myliobatidoidei. Atémesmo em Rhinoptera javanica, descrita e ligurada por NisHiD^ (1990: 61, fig. 39A), não há formação da fenestra.

\section{CONCLUSÕES}

Este estudo permitiu o estabelecimento de diferenças interespecificas, intraespecíficas e em diferentes estágios ontogenéticos no escápulo-coracóide e na cartilagem sinarcual cérvico-torácica de Rhinoptera brasiliensis e $R$. bonasus do !itoral brasileiro.

Quanto ao escápulo-coracóide, observou-se que em R. brasiliensis o forâmen escapular apresenta maior diâmetro, assim como a lenestra póstero-dorsal é maior, porém apenas quando comparados espécimens com maior largura de disco; em $R$. boncisus, a ponte das fenestras anteriores é mais espessa, há uma maior reentrância entre o mesocôndilo e o metacôndilo, sendo este mais estreito e ligeiramente mais longo. 
Já na sinarcual, verificou-se, dentre outras características, que em $R$. brasiliensis a porção superior da ponte ântero-escapular é encoberta totalmente pelo suporte lateral, estando exposta em $R$. bonasus. Na maioria dos espécimens de $R$. brasiliensis, observou-se a presença de mais de um par de forâmens basais, enquanto em $R$. bonasus, apenas um par. Observou-se também uma assimetria no posicionamento dos forâmens, mais acentuada em $R$. bonasus, bem como uma variação em relação às extensões laterais, mais marcante em $R$. brasiliensis. Há, ainda, uma variação individual no número destes forâmens. O exemplar AC.DBA V.UERJ. 926 ( $R$. bonasus) apresenta apenas um forâmen basal; AC.DBAV.UERJ. 770 e AC.DBAV.UERJ. 887 (ambos $R$. brasiliensis), três forâmens; e o exemplar AC.DBA V.UERJ. 914 (R. brasiliensis), de pequenas dimensões ( $\mathrm{LD}=422 \mathrm{~mm}$ e $\mathrm{CD}=240 \mathrm{~mm})$, dois forâmens basais.

Sendo assim, com mais este estudo, corrobora-se a validade de ambas as espécies de Rhinoptera para o Brasil.

\begin{abstract}
AGRADECIMENTOS. Agradecemos a Hugo Ricardo Secioso Santos, Paulo Marques Brito e Ulisses Leite Gomes pela leitura crítica e valiosas sugestões; a Alexander W.A. Kellner pela revisão do Abstract; à Aline Jardim da Fonseca pelas discussões sobre as estruturas anatômicas aqui estudadas; ao Hugo Ricardo Secioso Santos pelas ilustrações. Ao CNPq através de Bolsa de Aperfeiçoamento à A.F. A fonso e ao Convênio UERJ/FAPERJ pela bolsa de produtividade à V. Gallo.
\end{abstract}

\title{
REFERÊNCIAS BIBLIOGRÁFICAS
}

Bigelow, H.B. \& W.C. Scitroeder. 1953. Sawfishes, guitarfishes, skates and rays. In: Fishes of the Western North Atlantic. Mem. Sears Found. Mar. Res. 1 (2): 1-514.

BRITO, P.M. \& B. SERET. 1996. The new genus Iansan (Chondrichthyes, Rhinobatoidea) from the Early Cretaceous of Brazil and its phylogenetic relationships, p. 47-62. In: G. ARRATIA \& G. ViolIL (Eds). Mesozoic Fishes-Systematics and Paleoecology. München, Verlag Dr. Friedrich Pfeil, 576p.

Cavalcanti, M.J.; V. Gallo-Da-Silva \& A.F. Afonso. 1997. Identificação de Rhinoptera bonasus (Mitchill, 1815) e R. brasiliensis Müller \& Henle, 1841 (Chondrichthyes, Myliobatiformes, Rhinopteridae) pela análise de funções discriminantes. Acta Biol. Leopold. 19 (2): 205-215.

Compagno, L.J.V. 1973. Interrelationships of living elasmobranchs. In: P.H. GREenwoOd; R.S. MILES \& C. PATTERSOn (Eds). Interrelationships of fishes. Zool. Jour. Linn. Soc. 53 (Supl. 1): 15-61. . 1977. Phyletic relationships of living sharks and rays. Amer. Zool. 17 (2): 303-322.

Daniel, J.F. 1934. The elasmobranch fishes. Berkeley, Univ. California Press, 332p.

Gal.I.O-DA-SII.VA, V.; A.F. AFOnSO \& U.L. Gomf.S. 1997. Estudo taxonômico das espécies de Rhinoptera Cuvier, 1829 ocorrentes no litoral brasilciro. Parte I: Condroneurocrânio de exemplares jovens de Rhinoptera brasiliensis Müller \& Henle, 1841 e Rhinoptera bonasus (Mitchill, 1815) (Elasmobranchii, Myliobatiformes, Rhinopteridae). An. Acad. bras. Ci. 69 (4): 585-600.

Garman, S. 1913. The Plagiostomia. Mem. Mus. Comp. Zool. Harvard 36: 1-515.

Gomls, U.L.; C. Winklistlin \& W. SouZA-Lima. 1997. Estudo da cartilagem cérvico-torácica em Rajídeos (Batomorphii, Rajiformes) da região Sudeste do Brasil. An. Acad. bras. Ci. 69 (1): 95-107.

Hor.mGRFN, N. 1940. Studies on the head in fishes. Embryological, morphological and phylogenetical researches. Part I: Development of the skull in sharks and rays. Act. Zool. 21: 51-257.

. 1942. Studies on the head in fishes. An embryological, morphological and phylogenetical study. Part III: The phylogeny of elasmobranch fishes. Act. Zool. 23: 129-261.

JanVier, P. 1996. Early Vertebrates. New York, Oxford Univ. Press, 375p.

Revta bras. 
LLIBLLE, M.D. 1988. Revisión de metodos para estudios taxonomicos de rayas (Rajiformes, Rajidae) Gayana Zool. 52 (1-2): 15-93.

LUVEJUY, N.R. 1996. Systematics of myliobatoid elasmobranchs: with emphasis on the phylogeny and historical biogeography of neotropical freshwater stingrays (Potamotrygonidae: Rajiformes). Zool. Jour. Linn. Soc. 1996: 207-257.

Lund, R. \& E.D. Grogan. 1997. Relationships of the Chimaeriformes and the basal radiation of the Chondrichthyes. Rev. Fish Biol. Fisher. 7: 65-123.

MAISLY, J.G. 1984. Higher elasmobranch phylogeny and biostratigraphy. Zool. Jour. Linn. Soc. 82: 33-54.

- 1991. Santana Fossils: An illustrated Atlas. Neptune City, T.F.H. Publ. Inc., 459p.

MCEACIRAN, J.D. 1983. Results of the research cruises of FRV "Walther Herwig" to South America. LXI: Revision of the South Aınerican skate genus Psammobatis Günther, 1879 (Elasmobranchii, Rajiformes, Rajidae). Arch. Fischereiwiss. 34: 23-80.

- 1984. Anatomical investigations of the New Zealand skates Bathyraja asperula and B. spinifera with an eveluation of their classification within Rajoidei (Chondrichthyes, Rajiformes). Copeia 1984: 45-58.

McEaciran, J.D. \& L.J.V. Compagno. 1979. A further description of Gurgesiella furvescens, with comments on the interrelationships of Gurgesiellidae and Pseudorajidae (Pisces, Rajoidei). Bull. Mar. Sci. 29: 530-553.

MCEACIIRAN, J.D. \& T. MIYAKE. 1990. Phylogenetic interrelationships of skates: a working hypothesis (Chondrichthyes, Rajoidei), p. 285-304. In: Elasmobranchs as Living resources: ^dvances in the biology, ecology, systematics, and status of the lisheries. NOAA Tech. Rep., NMFS 90.

McEaciliran, J.D.; K.A. Dunn \& T. MiYake. 1996. Interrelationships of the batoid fishes (Chondrichthyes: Batoidea), p. 63-84. In: M.L.I. SIIASSNY; L.R. PARLNII \& G. DAVID JUHNSON (Eds.). Interrelationships of tishes. San Diego, Academic Press, 496p.

Mi:LoUk, M.A. 1949. On the relation between the vertebral column and the occiptal region of the chondrocranium in the selachii and its phylogenetics significances. Publ. Mar. Biol. Stat. Ghardaqa. 6: 45-51.

NisıIDA, K. 1990. Phylogeny of the suborder Myliobatidoidei. Mem. Fac. Fish. Hokkaido Univ. 37 (1/2): 1-108.

NisIIIDA, K. \& K. Nakaya. 1988. ^ new species of the genus Dasyatis (Elasmobranchii: Dasyatididae) from southem .Japan and lectotype designation of D. zugei. Japan Jour. Ichthyol. 35: 115-123.

Ørvig, T. 1962. Y a-t-il une relation directe entre les Arthrodires Ptyctodontides et les Holocephales? Problèncs actuels de Paleont. (Évol. des vertébrés). Colloques Inter. CNRS 104: 46-61.

PATtERSon, C. 1965. The phylogeny of the chimaeroids. Phill. Trans. R. Soc. 249B: 101-219.

REgan, C.T. 1906. ^ classification of the selachian fishes. Proc. Zool. London 1906: 722-758.

SAINT-Seine, M.P. 1949. Les poisson des calcaires lithographiques de Cerin ( $\wedge$ in). Nouv. Arch. Mus.

Hist. Nat. Lyon 2: 1-357.

SuIraI, S. 1992. Squalean phylogeny a new framework of "Squaloid" sharks and related taxa. Hokkaido, Hokkaido Univ. Press, 139p.

Si.va-Santos, R. 1968. A paleoictiof auna da Formação Santana-Euselachii. An. Acad. bras. Ci. 49 (4): $491-497$

Stensiö, E. 1969. Elasmobranchiomorphi, Placodermata, Arthrodires, p. 71-692. In: J. Piveteau (Ed.).

Traité de Paléontologic 4. Paris, Masson, Vol. 2. 790p.

Recebido em 13.1.2000, aceito em 24.IV.2001. 